\title{
CRÓNICA DEL SEXTO SEMINARIO HISPANO-ALEMÁN DE DERECHO PÚBLICO: LA UNIÓN EUROPEA EN EL CONTEXTO DEL BREXIT
}

\author{
ANTONIO MARTÍ DEL MORAL ${ }^{1}$ \\ Universidad de Jaén \\ amarti@ujaen.es \\ LOURDES DE LA TORRE MARTÍNEZ ${ }^{2}$ \\ Universidad de Jaén \\ lotorre@ujaen.es
}

Cómo citar/Citation

Martí del Moral, A. y Torre Martínez, L. de la (2017).

Crónica del Sexto Seminario Hispano-Alemán de Derecho Público:

la Unión Europea en el contexto del brexit.

Revista de Administración Pública, 202, 381-391.

doi: https://doi.org/10.18042/cepc/rap.202.13

Esta breve crónica refiere el desarrollo del 6o Seminario HispanoAlemán de Derecho Público que ha tenido lugar en Potsdam (Alemania) los días 23 y 24 de septiembre de 2016 con el título «A la vista de Brexit: la Unión Europea entre la rigidez y el cambio/Im Angesicht eines, Brexits: Die EU zwischen Beharren und Wandel», en el que, una vez más, se han debatido los principales problemas que afectan al funcionamiento de la Unión Europea. Ahora, en un escenario inédito y parcialmente caracterizado por la repercusión del brexit. Tales cuestiones se han analizado por profesores de distintas universidades alemanas, españolas y también — de enorme interés- británicas.

\footnotetext{
1 Profesor titular de Derecho Administrativo.

2 Profesora contratada doctora de Derecho Administrativo (acreditada PTU).
} 
El Seminario Hispano-Alemán —hagamos un breve recordatorio- surgió hace varios años gracias a la colaboración académica y personal entre $\mathrm{K}$. Stern (Universidad de Colonia), A. Jiménez-Blanco (Universidad Politécnica de Madrid y antes Granada y Jaén) y J. C. Pielow (Universidad de Bochum). Se han sucedido cinco Jornadas (Sevilla 2008, Bochum 2010, Baeza 2012, Colonia 2013, La Laguna-Tenerife 2015), de algunas de las cuales se ha dado cuenta anteriormente en las revistas especializadas, hasta la última en Potsdam.

La elección de los temas que han sido objeto de reflexión en los Seminarios apunta al interés inmediato de cada momento. El Seminario celebrado en Colonia en 2013, por ejemplo, versó sobre «La democracia en tiempos de crisis", abordó cuestiones novedosas de enorme impacto en el modo de configurar las normas y la aplicación del ordenamiento, así como en el funcionamiento de las instituciones públicas. Y el Seminario de 2016 en Potsdam se ha centrado, se insiste, en el análisis de las consecuencias y repercusiones a nivel europeo y nacional que ha producido la situación de la UE marcada por el brexit y el inicio (anunciado por el Gobierno británico) del procedimiento de retirada de Gran Bretaña, así como por el futuro de la UE, que pierde a la que es la tercera potencia económica.

La lengua de trabajo fue exclusivamente el alemán, a diferencia de las ocasiones anteriores. Debe indicarse además que el Seminario se realizó con el patrocinio y colaboración del Servicio Alemán de Intercambio Académico (DAAD).

Los dos días de trabajo se desarrollaron en torno a varias ponencias que permitieron abrir un debate con intervenciones de los asistentes. Avanzamos el programa y los ponentes y luego desarrollaremos el contenido de las intervenciones atendiendo a los aspectos que parecen más relevantes.

1. Introducción general de K. Stern.

2. Condiciones marco: jurídicas, políticas, sociales y otras de pensamiento (A.Weber, Osnabrück).

3. La regulación como legado británico a la Unión Europea (I. del Guayo, Almería).

4. Posiciones y sentimientos ante el brexit. Repercusiones a nivel nacional en Gran Bretaña (M. Hoorspol, Queen Mary College, British Institute, Londres), Alemania (S. Pieper, Bundespräsidalamt Berlin/Asesoría Jurídica de la Oficina del Presidente Federal) y España (M. M. Darnaculleta, Gerona).

5. La salida de la UE según el artículo 50 TUE: requisitos y modalidades (H. J. Blanke, Erfurt).

6. El déficit democrático en la Unión Europea (A. Marti, Universidad de Jaén, y L. de la Torre, Jaén). 
7. Resoluciones a tomar a corto plazo a nivel de la UE en relación con el mercado interior de la UE (J. C. Pielow) y en relación con la unión económica y monetaria: la aplicación en España del art. 126 TFUE (A. Jiménez-Blanco).

En estas líneas resulta imposible dar cuenta detallada de todas las cuestiones que se abordaron en el Seminario. No obstante, recordamos algunos de los aspectos más relevantes que allí se trataron.

El debate del primer día lo inició K. Stern, que hizo un repaso de la historia política y jurídica de Potsdam en el contexto europeo mencionando, entre otras cosas, algunos de los palacios y lugares (Palacio de Sanssouci) que han visto acontecimientos singulares de enorme trascendencia, entre otros destaca el reinado de Federico II de Prusia, en el que la nobleza se traslada a esa pequeña ciudad desde Berlín. Posteriormente muchos funcionarios gubernamentales fueron también a residir en Potsdam. Stern comentó la famosa Conferencia del 17 julio-2 agosto de 1945 en el Cecilienhof, donde los líderes aliados decidieron la división de Alemania y de Berlín, que marcó el desarrollo de la posguerra hasta que, con motivo de la reunificación alemana en 1990, Potsdam se convirtió en capital del estado de Brandeburgo.

Entrando en el primer bloque relativo a las condiciones, jurídicas, políticas, sociales y otros aspectos relativos a la mentalidad o modo de pensar colectivo, A. Weber analizó las consecuencias legales derivadas del referéndum británico. Se formularon preguntas básicas sobre la negociación de la salida de la Unión Europea (UE), y se respondió afirmando que el art. 50 TEU plantea muchas dudas que no admiten una solución completa a nivel europeo, porque también dependen del ordenamiento constitucional de cada Estado miembro. En este sentido, se convino la necesidad de mantener, frente al trato especial que ha caracterizado la situación de Gran Bretańa en la UE, la unidad para tutelar los intereses comunes de la UE. También el ponente examinó las consecuencias políticas que derivan del brexit y comentó las ideas defendidas por algunos movimientos populistas que piden un referéndum para la salida de la UE (Austria y Francia). Se planteó si la UE debe responder con una mayor integración o, por el contrario, ralentizar la armonización de ciertos sectores. Por último, Weber propuso medidas concretas para fortalecer la legitimidad democrática de la UE que dependen de la voluntad política de los protagonistas.

Del Guayo presentó el tema de la regulación económica como el legado principal del Reino Unido al ordenamiento jurídico de la UE, y explicó sus caracteres afirmando que es una alternativa conceptual a la intervención pública propia del derecho administrativo económico. La diferencia está en el punto de partida: la regulación presupone la primacía de los mercados y está dirigida a corregir sus fallos, mientras que la intervención administrativa implica que el Estado dirige los procesos económicos, pero trata de dejar un 
espacio de libertad a los individuos. La regulación y las agencias reguladoras independientes están hoy incorporadas al ordenamiento jurídico de la UE y de sus Estados miembros, precisamente por influencia de los sistemas jurídicos anglo-norteamericanos. La regulación de empresas —antes públicas y ahora privatizadas - ha sido una aportación destacada de Gran Bretaña a la reciente historia europea. Así pues, y en resumidas cuentas, la regulación nació a finales del siglo xx como regulación para la competencia, no en su contra.

También apuntó que el uso del vocablo «regulación» para referirse a la intervención administrativa en la vida económica es novedoso en España y en otras partes del mundo. La tensión entre el saber (regulación) y el poder (políticas públicas) se ha agudizado durante la crisis económica, aunque en todo caso la gobernanza de los sistemas económicos exige una combinación de política y de regulación, entre — de nuevo- el poder y el saber. A su juicio, el resultado del brexit acentúa las diferencias entre la regulación económica y el derecho público económico, pero confia en que el genio político británico contribuirá a recomponer satisfactoriamente la situación.

Posteriormente se abordaron las diferentes posiciones y efectos del brexit, así como sus repercusiones a nivel nacional en Gran Bretańa, Alemania y España. En este sentido, M. Horspool (Universidad de Londres), que recientemente ha publicado European Union Law (9a edición, OPU, septiembre 2016), se extendió sobre la distribución del voto y el perfil de los votantes en el referéndum del 23 de junio, así como la situación social y jurídica tras el brexit.

En el debate se mencionaron diversas cuestiones sobre el procedimiento de retirada de la UE conforme al art. 50 TUE, desde el inicio del período de dos años de negociaciones para salir de la UE, así como la conveniencia de garantizar el acceso al mercado único y preservar los derechos laborales. Tambien se puso de manifiesto la ambigüedad del art. $50 \mathrm{TUE}$, que establece que un Estado puede dejar la UE «de acuerdo a sus propios requerimientos constitucionales», lo que ha llevado a un desacuerdo en el Reino Unido, que no cuenta con una Constitución escrita. Conviene apuntar incidentalmente que, tras el Seminario celebrado en septiembre, las dudas e incertidumbres que plantea el art. 50 TUE se han incrementado por el dictamen del Tribunal Superior británico de 3 de noviembre de 2016, que obliga al Gobierno a pedir autorización al Parlamento antes de iniciar el proceso de salida de la UE, si bien el propio Gobierno ha recurrido esta decisión ante el Tribunal Supremo (El País, 4 de noviembre de 2016).

Volviendo a la crónica del Seminario, intervino S. Pieper, que realizó una completa exposición sobre las repercusiones del brexit en Alemania. Destacó la sorpresa de ser la primera salida unilateral en la historia de la integración europea, por ello las negociaciones sobre la relación futura entre la UE y GB deben clarificar las diferencias que existen si se trata de un Estado miembro de 
la Unión o, por contra, un tercer Estado. El modo de negociación será importante para impedir que la salida de los británicos devenga en un precedente atractivo para otros Estados miembros, alentados por falsas expectativas que pongan en crisis la integración.

Para Alemania, la definición de la futura relación con GB resulta muy significativa, porque es uno de los socios comerciales más importantes en la UE. En cualquier caso, es de esperar que las relaciones en cuestiones de seguridad exterior y relaciones internacionales (OTAN) continúen de forma estrecha.

En el debate se puso de manifiesto que la integración europea tendría que profundizar más en sentido cualitativo y menos cuantitativo. El brexit obliga a que Europa avance con éxito y no favorezca, directa o indirectamente, otras pretensiones de salida. En Alemania todos los partidos políticos consideran que el 23 de junio fue un día negro para Europa, salvo para un partido político (Alternativa para Alemania). En definitiva, en diversas intervenciones en el Seminario los asistentes afirmaron que el brexit marca un punto de inflexión en la integración europea y descubre de una nueva renacionalización en el planteamiento de los problemas políticos, económicos y sociales.

También se recordó que la crisis política (no solo económica) en Europa que ha supuesto el brexit se arrastra desde el rechazo de varios Estados miembros al Proyecto de Constitución Europea (2004). Se mencionaron varios factores importantes al respecto. La crisis de los Estados deudores en los Estados miembros del sur y la crisis de los refugiados en el Mediterráneo fortalecen una tendencia a ver las cosas desde el punto de vista nacional. También, sucede que parte de las élites políticas (populismos nacionalistas y antieuropeos) en los Estados miembros y también sectores no pequeños de la población han asumido la idea de que es mejor que los Estados vayan solos sin la UE.

Todo ello trae causa de motivos de muy variada naturaleza, que se desmenuzaron en el Seminario y se pueden recapitular de la forma siguiente:

1. Los países miembros del Este, después de la caída del Muro de Berlín, tenían puestas grandes esperanzas en la UE con el fin de obtener ventajas económicas. Sin embargo, Pieper recordó que estos Estados no eran conscientes (o no quisieron serlo) de la renuncia a la soberanía que está vinculada a la pertenencia a la UE, y por ello se aprecia un fortalecimiento intenso de lo nacional (Hungría, Polonia). En este sentido, Jiménez-Blanco añadió que a la frontera de la UE que antes se establecía en la línea norte-sur, se está añadiendo la oeste-este.

2. En el ámbito de la política europea se encuentra un creciente paso de discusiones y decisiones desde las instituciones europeas hacia las estructuras gubernamentales. Así sucede en las medidas de rescate en la crisis de los Estados deudores y en la crisis de refugiados. 
3. La crisis actual en la UE no es una crisis global de la integración europea, pues el sistema supranacional funciona en la mayoría de los ámbitos bien y casi sin queja. Ahora bien, si se pretende abordar la crisis, es precisa una evaluación clara de la necesidad de reforma y de su sentido. La solución que Europa necesita no atiende tanto a un cambio general de la arquitectura institucional como a la pérdida de la mentalidad europea de los ciudadanos y de parte de los líderes políticos en los Estados miembros. En el debate sobre estas ideas, Ibler (U. Konstanz) y Weber aludieron también al papel de los medios de comunicación y a la necesidad de organizar canales oficiales de la UE que formen opinión pública de forma coherente.

La siguiente ponencia en el Seminario se centró en el análisis de los requisitos previstos en el art. 50 TUE para la salida de la UE. Al respecto, Blanke destacó la importancia de los principios de subsidiariedad, atribución y proporcionalidad en la toma de decisiones europea (art. 5 TUE y Protocolo sobre la aplicación de los principios de subsidiariedad y de proporcionalidad). Si bien se ha reforzado el principio de subsidiariedad mediante los nuevos mecanismos en el Tratado de Lisboa, Blanke consideró insuficiente el control que sobre los poderes de la UE ha ejercido la jurisprudencia del Tribunal de Justicia de la UE hasta la fecha.

Para terminar el primer día del Seminario se presentaron dos ponencias sobre el déficit democrático de la UE y un debate posterior con los asistentes. En la primera ponencia, De la Torre señaló que el concepto de déficit democrático - el reproche, para entendernos - fue utilizado inicialmente para criticar la transferencia de los poderes legislativos de los Gobiernos nacionales al Consejo de Ministros de la UE. Esto llevó a la conveniencia de crear un Parlamento Europeo elegido directamente por los ciudadanos, que se ha transformado de un órgano consultivo en otro bien distinto y colegislador (art. 14 TUE). Así pues, resulta inaceptable la idea extendida por el populismo de que la única legitimación es la que procede de la democracia directa con exclusión de la representativa, que por otra parte acepta expresamente el Tratado de Lisboa (art. 10.1 TUE).

En cuanto al origen de las instituciones, se recordó que no es comparable la creación de cada uno de los Estados miembros, a partir de un acto explícito de un poder constituyente, y la propia de la UE, que representa un organismo supranacional al que se atribuyen competencias desde los Estados miembros para la realización de determinadas tareas con el fin puesto en «una unión cada vez más estrecha entre los pueblos de Europa» (preámbulo del TUE).

En la segunda ponencia sobre el déficit democrático, Martí recordó que en el inicio de las instituciones europeas el esquema responde a una legitimidad indirecta radicada en la presencia de los representantes de los Estados 
miembros elegidos por sus Ejecutivos nacionales, y se rompe en 1979 cuando se pasa a elegir al Parlamento Europeo por sufragio universal y directo, de modo que se reconoce la existencia de un «demos» a nivel europeo. En consecuencia, se aprecia un choque en el ámbito de legitimidad entre la Comisión Europea (y el Consejo) y el Parlamento Europeo. Posteriormente, los Tratados de Lisboa de 2009 han fortalecido las tareas del Parlamento Europeo otorgando, entre otras, la potestad de nombrar al presidente de la Comisión Europea, a propuesta del Consejo Europeo en función de los resultados de las elecciones, por mayoría cualificada. En todo caso, se constata un distanciamiento progresivo entre los ciudadanos y el Parlamento Europeo, acreditado por la baja participación en las elecciones europeas en las últimas convocatorias. Además, han aparecido otros factores que dificultan el proceso de integración y alteran las relaciones sociales: la heterogeneidad y pluralidad de la sociedad, así como la incapacidad de los Parlamentos y Gobiernos nacionales de representar a la misma, conducen a la falta de identificación de los ciudadanos con los poderes políticos, incluso a las escalas más bajas y cercanas. A todo ello se añade la proliferación de Estados deudores e incumplidores irredentos de los límites de déficit y deuda pública fijados por los Tratados, cuyas consecuencias no resultan fácil de explicar y aceptar por los ciudadanos.

También se puso de manifiesto que, a pesar de las modificaciones de los Tratados que persiguen reforzar la democracia europea (arts. 11.3 y 4 TUE, sobre la iniciativa ciudadana), no se puede ocultar la insuficiencia del régimen actual y la necesidad de encontrar nuevos cauces para mejorar el funcionamiento de la UE. Se comentó que para superar el déficit democrático no hay una solución única ni fórmulas mágicas que se puedan adoptar con carácter permanente, sino que hay que ir avanzando «step by step», como es propio del método de integración europeo desde los inicios. Todo ello se entiende porque la democracia en la UE no puede consistir en una repetición del mismo esquema de la democracia en los Estados nacionales; la UE no es un Estado sino un organismo supranacional que se diseña desde el origen con caracteres peculiares y determinados fines comunes.

Ni que decir tiene que se disertó mucho sobre la nueva situación de los partidos y movimientos políticos caracterizada por el populismo (de izquierdas o derechas), que promueve el desmantelamiento o abiertamente la destrucción de las instituciones europeas, tirando por la borda lo que ha sido un éxito sin precedentes en la historia de los países europeos. El momento histórico que marca la "crisis existencial» de la UE, como reconoce el presidente Juncker de la Comisión Europea, exige promover reformas concretas que consigan avances rápidos que sean perceptibles por los ciudadanos.

Hasta aquí hemos comentado el contenido del primer día del Seminario. El día 24 de septiembre se centró el debate en las posibles soluciones a tomar 
a corto plazo a nivel de la UE. Jiménez-Blanco analizó la unión económica y monetaria y la aplicación en España del art. 126 del TFUE. Comenzó recordando que España ha tenido, desde, al menos, el siglo XVI, una deuda pública altísima como una enfermedad crónica. Y además los acreedores han sido siempre extranjeros: primero (con Carlos I y Felipe II) alemanes y luego (con los Austrias menores, ya en el siglo XVII) genoveses. De ahí surge la noción de «deuda perpetua».

En 2001, explicó, se produce la entrada en el club de la moneda única, que tiene unas reglas que significan justo lo contrario a lo que se venía haciendo: no hay posibilidad de devaluación (pérdida de la soberanía monetaria formal, por así decir) y se considera de la inflación como un mal a combatir. Eso implica un cambio total, del que la sociedad española (y por supuesto la clase política) no quiso darse cuenta. Desde luego, los gobernantes de la época no se tomaron la molestia de explicar nada. Ello no se mostró especialmente problemático en los años de bonanza (2001-2008), que además en España fueron muy acentuados y permitieron una recaudación tributaria suficiente para compensar los incrementos de gasto público. En ese contexto expansivo también permanecieron ocultas las deficiencias estructurales de la gobernanza económica que se diseñó para el euro en Maastricht en 1993. En lo que nos concierne, era el art. 104 del TCE (actual art. 126 del TFUE). La crisis de 2008-2009 lo cambió todo y el autor de las normas (en España y en Europa) no ha tenido más remedio que acabar tomando nota. Se han adoptado medidas para mejorar la gobernanza de la unión monetaria, en el sentido de centralizar las decisiones. Aunque, con toda probabilidad, de manera tardía e insuficiente, porque cada país sigue siendo un mundo. Los Gobiernos están muy temerosos ante su respectiva opinión pública.

El ponente comentó la Decisión del Consejo de 12 de julio de 2016, declarativa del incumplimiento de España, "por la que se establece que Espańa no ha tomado medidas eficaces para seguir la Recomendación de 21 de junio de 2013 del Consejo» y la necesidad de aplicar las medidas correctoras del apartado 11 del art. 126 del Tratado, con multa como «última ratio». En definitiva, indicó que hay que mejorar nuestro diseño institucional y sobre todo (no se dice, aunque constituye el requisito mayor) la mentalidad y los modos de actuar de la clase política española, y seguramente no solo de ella.

También consideró la reforma del art. 135 de la Constitución española, modificado en 2011 bajo la presión agobiante de Bruselas y las consecuencias de este cambio constitucional. La redacción final del precepto es todo menos implacable por dos motivos. Primero, el mandato prohibitivo del déficit solo se refiere al estructural (noción que no se define) y además no es absoluto, sino que se fija por reenvío a lo que en cada momento fijen unas autoridades europeas. Y segundo, porque todo queda diferido al año 2020. Así pues, la situa- 
ción de la Hacienda pública no se va a poner en orden hasta que los acreedores pasen a exigir mucho más dinero en concepto de interés. Hasta entonces, las normas, incluso las del más alto rango, pueden decir cuanto quieran que todo va a seguir igual. Más y más deuda.

La última ponencia del Seminario fue presentada por Pielow y versó sobre las medidas a adoptar por la UE, a la vista del brexit, respecto al mercado interior. Comenzó explicando la importancia de la economía británica en Europa y se centró en las consecuencias negativas para el comercio exterior británico que depende fuertemente de las exportaciones dentro de la UE, así como los posibles escenarios para la salida de la UE a partir de un brexit duro, un brexit light o incluso ningún brexit en caso de un nuevo referéndum contrario o una decisión del Parlamento británico, que parece muy improbable.

En la mesa redonda posterior sobre el futuro del mercado interior, Pielow apuntó que esta creación ha sido, a lo largo de los últimos sesenta años, toda una success story, aunque existen problemas, contradicciones, incoherencias y ambigüedades que deben ser afrontadas con las medidas oportunas. Destacó que el campo de tensión se produce siempre cuando se pretende una mayor armonización e integración y por tanto afecta a la (restante) soberanía de los Estados miembros, así como a la existencia del «déficit democrático» de la UE. De otra parte, se recogieron las críticas continuas, sobre todo en Alemania, frente a regulaciones detalladísimas que muestran una «hiper-regulación» europea.

Además, se recordó que la UE no es «Estado» sino una «unión de Estados (soberanos)» según el Tribunal Constitucional Federal alemán, en la que rige el principio de atribución de competencias (art. 5.2 TUE), reforzado por los principios de la subsidiaridad y proporcionalidad (arts. 5.4 y 5 TUE). Sin embargo, se denunció por parte alemana un menoscabo paulatino de estos principios debido a la dinámica propia tanto de la legislación como de la labor ejecutiva a nivel europeo.

Se aludió a las ambigüedades en la evolución del derecho del mercado interior y se puso de relieve con tono de rechazo que se ha admitido una interpretación sumamente «abierta» de las competencias legislativas de la UE. En efecto, la competencia de aproximación y armonización según el art. 114 TFUE ha sido interpretada de forma amplísima por parte del TJUE con apoyo en el método del efecto útil (en caso de dudas rige aquella interpretación de las normas europeas que asegure lo mejor posible la eficacia práctica del derecho europeo). Un buen ejemplo es la jurisprudencia reiterada acerca de la Directiva sobre publicidad y patrocinio de los productos del tabaco, así como la Sentencia del TJUE de 4 de mayo de 2016 (C-358/14), que desestima un recurso de anulación interpuesto por Polonia contra varios preceptos de la Directiva 2014/40/UE del Parlamento Europeo y del Consejo, de 3 de abril de 
2014, sobre productos del tabaco y por la que se deroga la Directiva 2001/37/ CE. Esta importante Sentencia fue objeto de atención en la última mesa redonda del Seminario y por su interés conviene ahora reproducir parcialmente el contenido respecto al derecho aplicable al mercado interior.

La Sentencia afirma, por ejemplo, que «si bien la mera constatación de disparidades entre las normativas nacionales no basta para justificar la utilización del artículo 114 TFUE, no sucede lo mismo en caso de divergencias entre las disposiciones legales, reglamentarias o administrativas de los Estados miembros que puedan obstaculizar el ejercicio de las libertades fundamentales y afectar por ello directamente al funcionamiento del mercado interior...» (apartado 32). Y añade que "de reiterada jurisprudencia resulta igualmente que es posible recurrir al artículo 114 TFUE como base jurídica para evitar la aparición de futuros obstáculos a los intercambios comerciales derivados de la evolución heterogénea de las legislaciones nacionales, siempre que la aparición de tales obstáculos sea probable y que la medida de que se trate tenga por objeto su prevención...» (apartado 33). «Por otra parte, el Tribunal de Justicia ha declarado que, si se cumplen los requisitos para recurrir al artículo 114 TFUE como base jurídica, no puede impedirse al legislador de la Unión que se funde en esta base jurídica por el hecho de que la protección de la salud pública sea determinante en las decisiones que deben tomarse...» (apartado 34).

La Sentencia citada afirma también que «el artículo 168 TFUE, apartado 1, párrafo primero, preceptúa que, al definirse y ejecutarse todas las políticas y acciones de la Unión, ha de garantizarse un alto nivel de protección de la salud humana, al tiempo que el artículo 114 TFUE, apartado 3, exige de forma expresa que, en la armonización realizada, se garantice un nivel de protección elevado de la salud humana» (apartado 35). Luego añade: "cuando existan obstáculos a los intercambios o sea probable la aparición de futuros obstáculos, derivados del hecho de que los Estados miembros hayan adoptado o estén en trámite de adoptar, en relación con un producto o con una categoría de productos, medidas divergentes que puedan garantizar niveles de protección distintos e impedir, por ello, la libre circulación del producto o productos en la Unión, el artículo 114 TFUE faculta al legislador de la Unión para que intervenga adoptando las medidas adecuadas, que respeten, por una parte, lo previsto en el apartado 3 de dicho artículo y, por otra, los principios jurídicos mencionados en el Tratado FUE o establecidos por la jurisprudencia, en especial el principio de proporcionalidad» (apartado 36). Así pues, «el artículo 114 TFUE confiere al legislador de la Unión un margen de apreciación en cuanto a la técnica de aproximación más adecuada para lograr el resultado deseado, en especial en los ámbitos que se caracterizan por particularidades técnicas complejas» (apartado 68). 
Finalmente, la STJUE de 4 de mayo de 2016 desestimó el recurso de anulación interpuesto por Polonia contra la norma europea, recurso que pretendía demostrar que el objetivo de protección de la salud humana se habría podido alcanzar mejor a nivel nacional en lo que respecta concretamente a la prohibición de la comercialización de los productos del tabaco que contienen mentol, por considerar que los efectos de estos productos se circunscriben a un pequeño número de Estados miembros (apartado 121).

Todas estas cuestiones, y otras que por la brevedad de esta crónica no pueden mencionarse, fueron debatidas en el Seminario de Potsdam los días 23 y 24 de septiembre, conforme a un programa que tuvo la intensidad requerida para estos temas. Las aportaciones de los participantes demostraron una enorme capacidad crítica y — cada quien con su enfoque territorial, por supuesto- sensibilidad ante el reto de la integración europea en un nuevo escenario. Tampoco faltaron, por supuesto, algunas concesiones turísticas disfrutando de la magnífica ciudad, rica en lugares que han sido testigos de la historia alemana y europea. 
